\title{
Generalized Peterson's Rules in Fuzzy Natural Logic
}

\author{
Petra Murinová and Viktor Pavliska and Michal Burda \\ University of Ostrava, \\ Institute for Research and Applications of Fuzzy Modeling NSC IT4Innovations, \\ 30. dubna 2270103 Ostrava 1, Czech Republic, \\ petra.murinova@osu.cz, viktor.pavliska@osu.cz,michal.burda@osu.cz
}

\begin{abstract}
The main objective of this paper is to continue the study of generalized Peterson's syllogisms and to formally define Peterson's rules of distributivity, quantity and quality. While past results were based on mathematical definitions of generalized quantifiers the idea of this article is to take advantage of the position of quantifiers within g raded Peterson's square of opposition.
\end{abstract}

Keywords: Generalized intermediate quantifiers, Generalized Peterson's syllogisms, Generalized Peterson's rules.

\section{Introduction}

\subsection{Generalized syllogisms}

The theory of generalized syllogisms was studied by several authors as a generalization of classical Aristotle's syllogisms ([14]) and new forms of Peterson's syllogisms [16, 13], who proposed 69 new syllogisms with intermediate quantifiers. Intermediate quantifiers are special linguistic expressions, for example almost all, few, many, a large part of, which were introduced and deeply studied by Thompson [16] and later by Peterson in his book [13].

Thompson did not consider a possibility of two intermediate quantifiers in both premises. This work was later extended by Peterson in [13], where he proved another special group of so-called non-trivial syllogisms containing intermediate quantifiers in both premises. Verification of the validity of special group of syllogisms was performed using Venn diagrams. Peterson's extension was based on an idea to replace classical quantifier by an intermediate one in the four classical figures, which yielded in new 105 valid intermediate syllogisms. The typical example of a non-trivial intermediate syllogism is as follows:

\begin{tabular}{l}
$P_{1}:$ Almost all multinational companies \\
publish shares. \\
$P_{2}:$ Most multinational companies are on \\
the World Stock Markets. \\
\hline$C:$ Some multinational companies on \\
the World Stock Markets publish shares.
\end{tabular}

Later in the 20th century, several authors followed up this work and proposed several forms of syllogisms with generalized quantifiers. In 1985, L. Zadeh semantically analyzed a special class of syllogisms with intermediate quantifiers in both premises ([18]). Zadeh's work was later extended and elaborated by many authors. The extended syllogistic reasoning (see, e.g., $[1,15])$ adds new quantifiers. Other mathematical models of some generalized quantifiers and its corresponding syllogisms were suggested by, for example, Hájek, Pereira and others $[3,2,11,12]$.

The original Peterson's book inspired Novák to develop mathematical formal definitions of generalized quantifiers in the frame of Łukasiewicz fuzzy type theory (LFTT). In several papers $[10,8,9,5] 105$ basic syllogisms were syntactically proven and a general structure of generalized syllogisms was proposed and the question of syllogisms with more premises was discussed. These results demonstrate the power of that formalism in developing a formal model of natural human reasoning.

\subsection{Methods for the verification of syllogisms}

Below is a list of methods, which can be used for interpretation of classical as well as generalized syllogisms.

- Applying of Venn's diagram and to construct algebraic proof

- Using graded Peterson's square of opposition 
- To find formal (syntactical) proofs of corresponding syllogisms

- Using Aristotle's and Peterson's rules

The verification of the validity of 105 classical syllogisms with intermediate quantifiers using modified Venn's diagram was introduced in [13] in Section 3. Peterson interpreted main Aristotle's syllogisms and selected species of intermediate syllogisms. Another verification of validity of syllogisms is based on "Peterson's square" of opposition. A model of the Peterson's square in mathematical fuzzy logic was introduced in [7]. The authors showed that this square can be interpreted using the formalism of higher-order fuzzy logic (the fuzzy type theory).

The next method for a verification of validity of syllogisms, which will be mainly discussed in this paper, is based on applying of Aristotle's rules for Aristotle's syllogisms and Peterson's rules for syllogisms with intermediate quantifiers. There are many publications which present Aristotle's rules ([13]). In [13], Peterson analyzed syllogisms with intermediate quantifiers which form classical Peterson's square using "Peterson's rules". This approach is devoted to designing generalized Peterson's rules which will be used for a verification a validity of generalized syllogisms with intermediate quantifiers based on generalized Peterson's square of opposition (see [7]).

\subsection{Main goals}

In this paper we build on our previous article [5] in which we proposed an algorithm for generalized syllogisms with one middle formula based on generalized Peterson's rules. We inferred all the valid forms of syllogisms which fulfilled all Peterson's rules.

The main idea is to formally define main Peterson's rules (distributivity and quality) and formally prove that the derived rules of quantity can be inferred. The motivation for future is to extend this approach for generalized Peterson's syllogisms and to propose generalized Peterson's rules for $n$ quantifiers and $n$ premises.

\section{Preliminaries}

The main objective of this section is, at first, to recall Aristotle's and Peterson's square of opposition. Then we introduce Peterson's rules which can be used for the verification of the validity of Peterson's syllogisms with intermediate quantifiers. It is important to emphasize that the idea of applying of rules is based on the position of quantifiers within Aristotle's and Peterson's square of opposition, respectively. The position of individual quantifiers is related to distribution rules, which play a very important role in verifying the validity of logical syllogisms.

\subsection{Aristotle's square}

The Aristotle's square of opposition is constructed using basic properties stated in the diagram below:

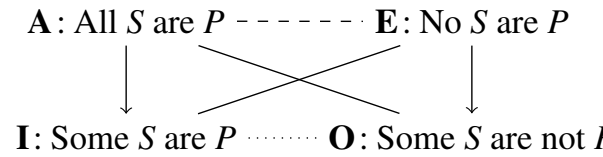

where the pairs $\mathbf{A}$ to $\mathbf{O}$ and $\mathbf{E}$ to $\mathbf{I}$ contradict each other, the two formulas of the top row $\mathbf{A}$ and $\mathbf{E}$ are contraries, the two formulas of the bottom row $\mathbf{I}$ and $\mathbf{O}$ are subcontraries, and the top of each column entails the formula under it. The precise definition of all the mentioned properties can be found in $[6,13]$. We can observe that both formulas $\mathbf{A}$ and $\mathbf{E}$ are universal and $\mathbf{I}$ and $\mathbf{O}$ are particular. Furthermore, the pair $\mathbf{A}$ and $\mathbf{I}$ is affirmative and $\mathbf{E}$ and $\mathbf{O}$ is negative.

\subsection{Peterson's square}

To work with Peterson's square of opposition means to assume intermediate quantifiers, which lay between classical ones.

Definition 2.1 (Proposition) Proposition is an expression in the form either

$$
Q(B, A) \quad \text { or } \quad Q(B, \neg A)
$$

where $Q$ is a $\langle 1,1\rangle$ quantifier according to the classification introduced in [4] and $A, B$ are terms. $Q(B, A)$ is an affirmative and $Q(B, \neg A)$ is a negative proposition.

Quantifier $Q$ specifies the quantity of elements satisfying $B$ that also satisfy $A$.

Peterson's square of opposition is formed by four main properties as follows:

Definition 2.2 - We say that two formulas are contradictory if in any model they cannot be both true, and they cannot be both false.

- We say that two formulas are contrary if in any model they cannot be both true, but both can be false.

- We say that two formulas are sub-contrary if in any model they cannot be both false, but both can be true. 
- A formula is sub-altern of another one, called a super-altern formula, if, in any model, it must be true if its super-altern is true. At the same time, the super-altern must be false if the sub-altern is false.

Below we introduce Peterson's square of opposition as an extension of the first Peterson's square with "Almost all" and "Many". The straight lines mark contradictories, the dashed lines contraries and the dotted lines sub-contraries. The arrows indicate the relation superaltern-subaltern.

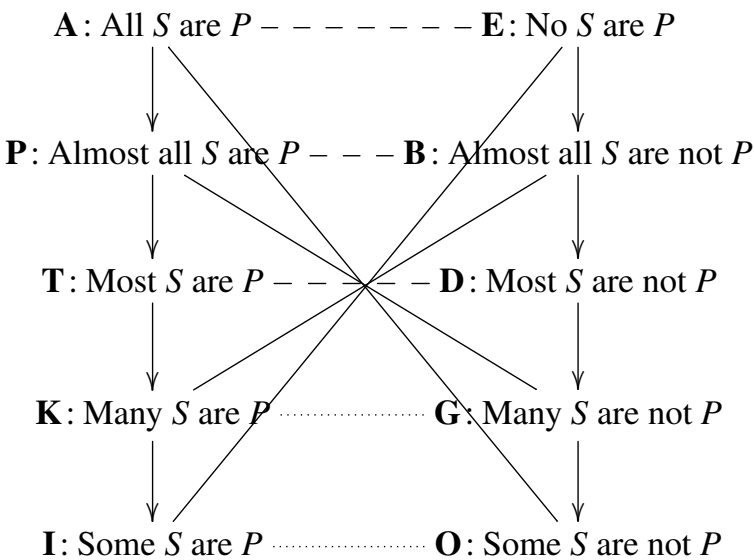

The implemented denoting of intermediate quantifiers stems from the position of individual quantifiers in the Peterson's square of opposition. Denoting AEIO for the Aristotle's square was explained in the previous subsection. The position of quantifier "Almost all" at a Predominant position implies being denoted as $\mathbf{P}$, and dually B for a negative form. Quantifier "Most" is denoted as $\mathbf{T}$ (majoriTy), and dually $\mathbf{D}$ for a negative form. Finally, quantifier "Many" has a common position and thus is denoted $\mathbf{K}$, and dually $\mathbf{G}$ for a negative form.

Peterson's square gives us an information if quantifiers are positive or negative, how "big" they are, etc. To verify validity/invalidity of syllogisms using generalized Peterson's rules does not mean to know the precise mathematical definitions of quantifiers, but we need to know the position of all the quantifiers inside the graded Peterson's square.

\subsection{Categorical syllogism}

Definition 2.3 (Syllogism) A syllogism is a triple $\left\langle P_{1}, P_{2}, C\right\rangle$ of three propositions. $P_{1}, P_{2}$ are called premises ( $P_{1}$ is major, $P_{2}$ is minor) and $C$ is called a conclusion. In these propositions, there appear a total of exactly three terms $S, P, M$, each of which is used exactly twice: term $S$ (subject) appears somewhere in $P_{2}$ and as the first term of $C$, term $P$ (predicate) appears somewhere in $P_{1}$ and as the second term of $C$; a term not present in the conclusion $C$ is called a middle term M. A syllogism is valid if the conclusion $C$ logically follows from premises $P_{1}$ and $P_{2}$.

Syllogisms with a single middle term yield the following four figures:

\begin{tabular}{cccc} 
Figure I & Figure II & Figure III & Figure IV \\
$Q_{1} M$ is $P$ & $Q_{1} P$ is $M$ & $Q_{1} M$ is $P$ & $Q_{1} P$ is $M$ \\
$Q_{2} S$ is $M$ & $Q_{2} S$ is $M$ & $Q_{2} M$ is $S$ & $Q_{2} M$ is $S$ \\
\hline$Q_{3} S$ is $P$ & $Q_{3} S$ is $P$ & $Q_{3} S$ is $P$ & $Q_{3} S$ is $P$
\end{tabular}

For the sake of simplicity, let us introduce a condensed expression of a syllogism, $\langle M P, S M \mid S P\rangle$, which corresponds to Figure-I. Typical examples of natural language linguistic expressions which form syllogisms with intermediate quantifiers are as follows:

- Several people during the COVID pandemic are stressed.

- Most people who live in area affected by heavy industry suffer from asthma.

- Many people after undergoing COVID have worsened chronic diseases.

The intermediate syllogism is obtained from any traditional syllogism (valid or not) replacing one or more of its formulas by formulas containing intermediate quantifiers. By non-trivial syllogism we denote the intermediate syllogism where both premises and also the conclusion consist of non-trivial ${ }^{1}$ intermediate quantifiers.

\section{Peterson's rules}

\subsection{Peterson's rules for Aristotle's syllogisms}

In [13], Peterson suggested modified Aristotle's rules for Aristotle's syllogisms. Below we recall Peterson's six rules of which only four, distribution and quality, turn out to be absolutely necessary. In [13], Peterson introduced four rules: two involving distribution and two involving quality. The other two rules of quantity were added, but these rules were inferred from the rules mentioned earlier. Differences between Aristotle's and Peterson's rules can be found in [5]. There are the mentioned rules:

\section{Rules of Distribution}

\footnotetext{
${ }^{1}$ By trivial intermediate quantifiers we means the quantifiers "All" and "Some".
} 
(R1) The middle formula must be distributed at least once.

(R2) No formula is distributed in the conclusion unless it is distributed in one premise.

To use these rules, we have to know what distribution is. We very briefly introduce that the subject formulas of universal formulas (A and $\mathbf{E}$ ) and the predicate formulas of negative formulas ( $\mathbf{E}$ and $\mathbf{O}$ ) are distributed. The other formulas are not distributed.

There is a deeper explanation. So, in the formula $\mathbf{A}$, "All S are P", the formula " $\mathrm{S}$ " is distributed, because the formula "All S are P" says something about every single $S$ - namely, that they are all $P$. In the formula E, "No S are P", both the formulas $S$ and $P$ are distributed, since you are making a claim about all S's, and all P's. Moving on to the formula I, "Some S are P", neither formula is distributed, and that's pretty easy to see. You are not making a claim about all of $S$, nor are you making a claim about all of $P$. In the formula $\mathbf{O}$, "Some $\mathrm{S}$ are not $\mathrm{P}$ ", the formula $P$ is distributed, because we have asserted something about all of $P$ namely, that all of $P$ falls outside at least one $S$.

\begin{tabular}{|c|c|c|c|c|}
\hline & Quantifier & Quantity & Quality & Distributed \\
\hline All S are P & A & universal & affirmative & S \\
\hline No S are P & E & universal & negative & S and P \\
\hline Some S are P & I & particular & affirmative & none \\
\hline Some S are not P & O & particular & negative & P \\
\hline
\end{tabular}

Example 3.1 (Rule (R1)) The rule (RI) explains a formal mistake in which a syllogism contains a middle formula that is not distribute in either premise. There is an example of invalid syllogism:

$$
\begin{array}{cl} 
& P_{1}: \text { All sharks are fish. } \\
\boldsymbol{A A A}-I I I & P_{2}: \text { All salmon are fish. } \\
\cline { 2 - 3 } & C: \text { All salmon are sharks. }
\end{array}
$$

Example 3.2 (Rule (R2)) The rule (R2) explains a formal mistake in which a syllogism contains a middle formula that is not distribute in either premise. There is an example of invalid syllogism:

$$
\begin{array}{cl}
\text { AOO-I } & P_{1}: \text { All horses are animals. } \\
\cline { 2 - 2 } & P_{2}: \text { Some dogs are not horses. } \\
\hline C: \text { Some dogs are not animals. }
\end{array}
$$

\section{Rules of Quality}

(R3) At least one premise is affirmative.

(R4) The conclusion is negative if and only one premise is.

The rule (R3) says that no conclusion draw from two negative premises. The rule (R4) says that negative premise requires a negative conclusion, and a negative conclusion requires a negative premise. Finally, if $a$ syllogism has a negative premise, it must have a negative conclusion, and vice versa.

Example 3.3 (Rule (R3)) There is an example of negative syllogism:

$$
\begin{aligned}
& \text { EOI-I } P_{1}: \text { No birds are mammals. } \\
& P_{2}: \text { Some dogs are not birds. } \\
& \hline C: \text { Some dogs are birds. }
\end{aligned}
$$

Example 3.4 (Rule (R4)) Similarly, the rule (R4) says that any syllogism having exactly one negative formula is invalid. There is an example of invalid syllogism:

$$
\begin{array}{cl}
\text { AOI-I } & P_{1}: \text { All crows are birds. } \\
& P_{2}: \text { Some wolves are not crows. } \\
\cline { 2 - 2 } \text { C: Some wolves are birds. }
\end{array}
$$

Thus, a negative conclusion cannot follow from positive premises.

\section{Rules of Quantity}

(R5) At least one premise is universal.

(R6) If either premise is particular, the conclusion is particular.

Example 3.5 (Rule (R5)) There is an example of invalid syllogism:

$$
\begin{array}{ll} 
& P_{1}: \text { Some jokes are funny. } \\
\text { III-III } & P_{2}: \text { Some jokes are old. } \\
\cline { 2 - 2 } \text { C : Some old jokes are funny. }
\end{array}
$$

Example 3.6 (Rule (R6)) There is an example of invalid syllogism:

$$
\begin{array}{ll}
P_{1}: \text { Some jokes are funny. } \\
\text { III-I } & P_{2}: \text { All jokes are old. } \\
\hline C: \text { All old jokes are funny. }
\end{array}
$$

\subsection{Peterson's rules for intermediate syllogisms}

The main objective of this section is to recall Peterson's rules for the verification of the validity of intermediate syllogisms.

We can notice that the rules (R2), (R3) and (R4) remain unchanged. The first distribution rule is reformulated with respect to the number of intermediate quantifiers. Furthermore, an important role is played by the quantifier, which determines majority, which is used in the rule (R5). 


\section{Rules of Distribution}

(R1) In a valid syllogism, the sum of distribution indices for the middle formula must exceed 5.

(R2) No formula may be more nearly distributed in the conclusion than it is in the premises.

Let us, for example, recall that the sum of distribution of valid syllogisms must exceed 5, which ensures nonempty intersection of domains if the middle formulas are on the position of subject. For an explanation related to other figures see [13].

The rules o quality remain unchanged.

\section{Rules of Quality}

(R3) At least one premise must be affirmative.

(R4) The conclusion is negative if and only if one of the premises is negative.

Example 3.7 (Rule (R3)) The rule (R3) says that no conclusion draw from two negative premises. There is an example of negative syllogism:

\section{$P_{1}:$ No fish are mammals. \\ EDD $-I \frac{P_{2}: \text { Most dogs are not fish. }}{C: \text { Most dogs are not mammals. }}$}

Example 3.8 (Rule (R4)) Similarly, the rule (R4) says that any syllogism having exactly one negative formula is invalid. There is an example of invalid syllogism:

$P_{1}:$ All crows are birds.

AOI-III $\frac{P_{2}: \text { Some wolves are not crows. }}{C: \text { Some wolves are birds. }}$

\section{Rules of Quantity}

(R5) At least one premise must have a quantity of majority ( $\mathbf{T}$ or $\mathbf{D}$ ) or higher.

(R6) If any premise is non-universal, the the conclusion must have a quantity that is less than or equal to that premise.

\section{Generalized Peterson's rules}

The main objective of this section and also of this paper is to propose mathematical definitions of Peterson's rules for intermediate quantifiers. We need to know if the quantifier is affirmative or negative ${ }^{2}$. Furthermore, we need to know a position of quantifier. It means to

\footnotetext{
${ }^{2}$ If we will say that the quantifier is positive then also the corresponding premise is positive. Similarly for the negative quantifier.
}

define the position inside of the generalized Peterson's square. Recall that if we work with five basic Peterson's quantifiers we speak about five positions as follows: universal, predominant, majority, common, particular. Finally, we want to analyze the position of the middle formula in the corresponding syllogisms. So, we continue with new functions which represent above mentioned properties.

A set of all considered quantifiers will be denoted by 2. For Peterson's framework of generalized intermediate syllogisms, we have $2=$ \{"all", "almost all", "most", "many", "some" $\}$. Each quantifier $Q \in \mathcal{Q}$ must have assigned a quantity $(Q)$ which is explained in the subsequent definition.

Definition 4.1 (Quantity) Let $Q$ be a quantifier. We say that

(a) $0<$ quantity $(Q) \leq 1$ for all $Q \in \mathscr{Q}$;

(b) quantity $\left(Q_{1}\right) \leq$ quantity $\left(Q_{2}\right)$ iff for $Q_{1}, Q_{2} \in \mathcal{Q}$, proposition $Q_{1}(B, A)$ is superaltern of proposition $Q_{2}(B, A)$;

(c) quantity $(Q)>0.5$ iff for $Q \in \mathcal{Q}$, proposition $Q(B, A)$ is contrary to $Q(B, \neg A)$;

(d) quantity $\left(Q_{1}\right)+$ quantity $\left(Q_{2}\right)>1$ if $Q_{1}(B, A)$ and $Q_{2}(B, \neg A)$ form a contradictory pair.

For Peterson's framework we can set (see [17])

- quantity("all") =1,

- quantity("almost all") $=p$,

- quantity ("most") $=t$

- quantity("many") $=k$,

- quantity("some") $=\varepsilon$

such that

(a) $0<\varepsilon<k<0.5<t<p<1-\varepsilon<1$,

(b) $k+p>1$,

(c) $t+k \leq 1$.

The property (a) guarantees a monotonous position of quantifies in Peterson's square. Specially, the condition $k<0.5<t<p$ means that $\mathbf{P}, \mathbf{B}$ have predominant position and $\mathbf{T}, \mathbf{D}$ have majority position. Moreover all positive as well as negative quantifiers $\mathbf{A}, \mathbf{E}, \mathbf{P}, \mathbf{B}, \mathbf{T}, \mathbf{D}$ are preponderance quantifiers while $\mathbf{K}, \mathbf{G}, \mathbf{I}, \mathbf{O}$ are not. 
Table 1: An overview of all properties related to the Peterson's rules

\begin{tabular}{|c|c|c|c|c|}
\hline & Quantifier & Quantity & Quality & Distributed \\
\hline All S are P & A & universal & affirmative & DI5:subjects, DI1:predicates \\
\hline No S are P & E & universal & negative & DI5:subjects, DI5:predicates \\
\hline Almost all S are P & P & predominant & affirmative & DI4:subjects, DI1:predicates \\
\hline Almost all S are not P & B & predominant & negative & DI4:subjects, DI5:predicates \\
\hline Most S are P & T & majority & affirmative & DI3:subjects, DI1:predicates \\
\hline Most S are not P & D & majority & negative & DI3:subjects, DI5:predicates \\
\hline Many S are P & K & common & negative & DI2:subjects, DI1:predicates \\
\hline Many S are not P & G & common & negative & DI2:subjects, DI5:predicates \\
\hline Some S are P & I & particular & affirmative & DI1:subjects, DI1:predicates \\
\hline Some S are not P & O & particular & negative & DI1:subjects, DI5:predicates \\
\hline
\end{tabular}

The condition (b) express the property of a contradictory of the pair $[\mathbf{P}, \mathbf{G}]$ as well as of $[\mathbf{B}, \mathbf{K}]$ while the condition (c) express the fact that $[\mathbf{K}, \mathbf{D}]$ and $[\mathbf{G}, \mathbf{T}]$ do not constitute a contradictory pair.

For the sake of brevity, we put quantity $(\mathscr{P})=$ quantity $(Q)$, for any proposition $\mathscr{P}=Q(.,$.$) .$

Definition 4.2 (Signum) The signum of proposition $\mathscr{P}$ is defined as

$$
\operatorname{signum}(\mathscr{P})= \begin{cases}1 & \text { if } \mathscr{P} \text { is affirmative } \\ 0 & \text { if } \mathscr{P} \text { is negative. }\end{cases}
$$

Definition 4.3 (Distribution) The distribution of term $T$ in proposition $\mathscr{P}, \operatorname{dist}(T, \mathscr{P})$, equals to

1. quantity $(Q)$ if $\mathscr{P}=Q(T, X)$;

2. $\varepsilon$ if $\mathscr{P}=Q(X, T)$ and $\mathscr{P}$ is affirmative;

3. 1 if $\mathscr{P}=Q(X, T)$ and $\mathscr{P}$ is negative.

Below we introduce all the six Peterson's rules of distributivity, quality and quantity defined using generalized logical operations.

Definition 4.4 (Peterson's rules) Let $\otimes \quad b e$ Łukasiewicz t-norm, $\wedge$ be Gödel t-norm (minimum), $\vee$ be Gödel t-conorm (maximum), $\mathscr{S}=\left\langle P_{1}, P_{2}, C\right\rangle$ be a syllogism such that $S$ is the first term of conclusion $C, P$ is the second term of conclusion $C$ and $M$ is the middle term.

\section{Rules of Distribution}

(R1) $\operatorname{dist}\left(M, P_{1}\right) \otimes \operatorname{dist}\left(M, P_{2}\right)>0$;

$(R 2 a) \operatorname{dist}(S, C) \leq \operatorname{dist}\left(S, P_{2}\right)$;

(R2b) $\operatorname{dist}(P, C) \leq \operatorname{dist}\left(P, P_{1}\right)$;

2. Rules of Quality

$(R 3) \operatorname{signum}\left(P_{1}\right) \vee \operatorname{signum}\left(P_{2}\right)=1$;

$(R 4) \operatorname{signum}\left(P_{1}\right) \wedge \operatorname{signum}\left(P_{2}\right)=\operatorname{signum}(C)$;

3. Rules of Quantity
(R5) quantity $\left(P_{1}\right) \vee$ quantity $\left(P_{2}\right)>0.5$;

(R6) quantity $\left(P_{1}\right) \wedge$ quantity $\left(P_{2}\right) \geq$ quantity $(C)$.

Theorem 4.5 The syllogism $\mathscr{S}$ is valid if the rules of distributivity and quality are satisfied.

Lemma 4.6 Let rules (R1-R4) be satisfied. Then the rule (R5) holds.

Proof 4.7 The proof will be constructed by analysis of the following cases:

\section{- Figure-I}

$$
\begin{aligned}
& Q_{1} M P \quad Q_{1} M \neg P \quad Q_{1} M \quad P \\
& \frac{Q_{2} S M}{Q_{C} S P} \quad \frac{Q_{2} S M}{Q_{C} S \neg P} \quad \frac{Q_{2} S \neg M}{Q_{C} S \neg P}
\end{aligned}
$$

Let $P_{1}=Q_{1}(M, P)$ or $P_{1}=Q_{1}(M, \neg P)$ and $P_{2}=$ $Q_{2}(S, M)$. Then $\operatorname{dist}\left(M, P_{1}\right)=$ quantity $\left(Q_{1}\right)$ and $\operatorname{dist}\left(M, P_{2}\right)=\varepsilon$. From the assumed validity of $(R 1)$ we know that

$$
\text { quantity }\left(Q_{1}\right)+\varepsilon>1,
$$

from which we obtain quantity $\left(Q_{1}\right)>1-\varepsilon>0.5$, which again yields (R5). Let $\langle M P, S \neg M \mid S \neg P\rangle$. This situation cannot occur because the rule $(R 2 b)$ is violated.

\section{- Figure-II}

$$
\begin{aligned}
& Q_{1} P M \quad Q_{1} P \neg M \quad Q_{1} P \quad M \\
& \frac{Q_{2} S M}{Q_{C} S P} \quad \frac{Q_{2} S \quad M}{Q_{C} S \neg P} \quad \frac{Q_{2} S \neg M}{Q_{C} S \neg P}
\end{aligned}
$$

The first form cannot occur because the (RI) is violated.

Let $P_{1}=Q_{1}(P, \neg M)$ or $P_{1}=Q_{1}(P, M)$ and $P_{2}=$ $Q_{2}(S, M)$ or $P_{2}=Q_{2}(S, \neg M)$ and $C=Q_{3}(S, \neg P)$. Then $\operatorname{dist}\left(P, P_{1}\right)=$ quantity $\left(Q_{1}\right)$ and $\operatorname{dist}(P, C)=1$. Using the rule $(R 2 b)$ we obtain that quantity $\left(Q_{1}\right)=1$ from which it follows (R5).

\section{- Figure-III}




$$
\begin{aligned}
& Q_{1} M P \quad Q_{1} M \neg P \quad Q_{1} M P \\
& \frac{Q_{2} M S}{Q_{C} S P} \quad \frac{Q_{2} M S}{Q_{C} S \neg P} \quad \frac{Q_{2} M \neg S}{Q_{C} S \neg P}
\end{aligned}
$$

Let $\langle M P, M S \mid S P\rangle,\langle M \neg P, M S \mid S \neg P\rangle,\langle M P, M \neg S \mid S \neg P\rangle$ be possible forms. Let $P_{1}=Q_{1}(M, P)$ or $P_{1}=$ $Q_{1}(M, \neg P)$ and $P_{2}=Q_{2}(M, S)$. Then $\operatorname{dist}\left(M, P_{1}\right)=$ quantity $\left(Q_{1}\right)$ and dist $\left(M, P_{2}\right)=$ quantity $\left(Q_{2}\right)$. Using the rule $(R 1)$ we obtain

$$
\text { quantity }\left(P_{1}\right)+\text { quantity }\left(P_{2}\right)>1
$$

and therefore

$$
\text { quantity }\left(P_{1}\right) \vee \text { quantity }\left(P_{2}\right)>0.5 \text {. }
$$

The form $\langle M P, M \neg S \mid S \neg P\rangle$ cannot occur because the rule $(R 2 b)$ is violated.

\section{- Figure-IV}

$$
\begin{array}{lll}
Q_{1} P M & \begin{array}{l}
Q_{1} P \neg M \\
Q_{2} M S
\end{array} & Q_{2} M S \\
Q_{C} S P & Q_{C} S \neg P & Q_{2} M \neg S \\
\hline Q_{C} S \neg P
\end{array}
$$

Let $P_{1}=Q_{1}(P, M)$ and $P_{2}=Q_{2}(M, S)$ or $P_{2}=$ $Q_{2}(M, \neg S)$. Then dist $\left(M, P_{1}\right)=\varepsilon$ and $\operatorname{dist}\left(M, P_{2}\right)=$ quantity $\left(Q_{2}\right)$. Then using the rule $(R 1)$ we have

$$
\text { quantity }\left(Q_{2}\right)+\varepsilon>1
$$

which fulfills (R5).

Let $P_{1}=Q_{1}(P, \neg M), \quad P_{2}=Q_{2}(M, S)$ and $P_{3}=$ $Q_{3}(S, \neg P)$. Then $\operatorname{dist}\left(P, P_{1}\right)=$ quantity $\left(Q_{1}\right)$ and $\operatorname{dist}(P, C)=1$. Hence using the rule $(R 2 b)$ it follows that quantity $\left(Q_{1}\right)=1$ which yields (R5).

Lemma 4.8 Let rules (RI-R4) be satisfied. Then the rule (R5) holds.

Proof 4.9 The proof will be constructed by analysis of the following cases:

\section{- Figure-I}

$$
\begin{array}{lll}
Q_{1} M P & Q_{1} M \neg P & Q_{1} M P \\
Q_{2} S M & Q_{2} S M & Q_{2} S \neg M \\
\hline Q_{C} S P & Q_{C} S \neg P & \frac{Q_{C} S \neg P}{Q_{C}}
\end{array}
$$

Let us assume $P_{1}=Q_{1}(M, P)$ or $P_{1}=Q_{1}(M, \neg P), P_{2}=$ $Q_{2}(S, M)$ and $C=Q_{3}(S, P)$ or $C=Q_{3}(S, \neg P)$. From the definition of distribution it follows $\operatorname{dist}(S, C)=$ quantity $(C)$ and $\operatorname{dist}\left(S, P_{2}\right)=$ quantity $\left(P_{2}\right)$. Using the rule $(R 2 a)$ we have

$$
\text { quantity }(C) \leq \text { quantity }\left(P_{2}\right) .
$$

Similarly, we know $\operatorname{dist}\left(M, P_{1}\right)=$ quantity $\left(P_{1}\right)$ and $\operatorname{dist}\left(M, P_{2}\right)=\varepsilon$. From $(R 1)$ we obtain

$$
\text { quantity }\left(P_{1}\right)+\varepsilon>1
$$

and hence quantity $\left(P_{1}\right)>1-\varepsilon>p$ which implies

$$
\text { quantity }(C) \leq \text { quantity }\left(P_{1}\right)
$$

Finally, from (1) and (2) we obtain quantity $(C) \leq$ quantity $\left(P_{1}\right) \wedge$ quantity $\left(P_{2}\right)$.

The last form violates the rule ( $R 2 b)$.

\section{- Figure-II}

$$
\begin{aligned}
& Q_{1} P M \quad Q_{1} P \neg M \quad Q_{1} P \quad M \\
& \frac{Q_{2} S M}{Q_{C} S P} \quad \frac{Q_{2} S M}{Q_{C} S \neg P} \quad \frac{Q_{2} S \neg M}{Q_{C} S \neg P}
\end{aligned}
$$

The first form cannot occur because the (RI) is violated.

Let $P_{1}=Q_{1}(P, \neg M)$ or $P_{1}=Q_{1}(P, M)$ and $P_{2}=$ $Q_{2}(S, M)$ or $P_{2}=Q_{2}(S, \neg M)$ and $C=Q_{3}(S, \neg P)$. We know that dist $\left(S, P_{2}\right)=q u a n t i t y\left(P_{2}\right)$ and dist $(S, C)=$ quantity $(C)$. From $(R 2 a)$ we have

$$
\text { quantity }(C) \leq \text { quantity }\left(P_{2}\right) .
$$

Furthermore, $\operatorname{dist}\left(P, P_{1}\right)=$ quantity $\left(P_{1}\right)$ and $\operatorname{dist}(P, C)=1$. Hence using the rule $(R 2 b)$

\begin{tabular}{|c|c|c|}
\hline$Q_{1} P M$ & $Q_{1} P \neg M$ & $\begin{array}{lll}Q_{1} & P & M \\
O_{2} & M & \neg\end{array}$ \\
\hline$Q_{2} M S$ & $Q_{2} M \quad S$ & $Q_{2} M \neg S$ \\
\hline$Q_{C} S P$ & $Q_{C} S \neg P$ & $Q_{C} S \neg P$ \\
\hline
\end{tabular}
quantity $\left(P_{1}\right)=1$ which means that

$$
\text { quantity }(C) \leq \text { quantity }\left(P_{1}\right) .
$$

We can conclude that from (3) and (4) we obtain the rule $(R 6)$.

\section{- Figure-III}

$$
\begin{aligned}
& Q_{1} M P \quad Q_{1} M \neg P \quad Q_{1} M P \\
& \frac{Q_{2} M S}{Q_{C} S P} \quad \frac{Q_{2} M S}{Q_{C} S \neg P} \quad \frac{Q_{2} M \neg S}{Q_{C} S \neg P}
\end{aligned}
$$

Let $P_{1}=Q_{1}(M, P)$ or $P_{1}=Q_{1}(M, \neg P)$ and $P_{2}=$ $Q_{2}(M, S)$. Then $\operatorname{dist}(S, C)=$ quantity $(C)$ and $\operatorname{dist}\left(S, P_{2}\right)=\varepsilon$. Then from $(R 2 a)$ it follows that quantity $(C) \leq \varepsilon$. Hence the rule (R6) is fulfilled.

The form $\langle M P, M \neg S \mid S \neg P\rangle$ cannot occur because the rule $(R 2 b)$ is violated.

\section{- Figure-IV}


Let $P_{1}=Q_{1}(P, M)$ and $P_{2}=Q_{2}(M, \neg S)$ and $C=$ $Q_{3}(S, \neg P)$. Then dist $\left(M, P_{2}\right)=$ quantity $\left(P_{2}\right)$ and $\operatorname{dist}\left(M, P_{1}\right)=\varepsilon$. Using the rule $(R 1)$ it follows quantity $\left(P_{2}\right)>1-\varepsilon$ and hence quantity $\left(P_{2}\right)=1$ which means

$$
\text { quantity }(C) \leq \text { quantity }\left(P_{2}\right)
$$

Furthermore, $\operatorname{dist}\left(P, P_{1}\right)=$ quantity $\left(P_{1}\right)$ and $\operatorname{dist}(P, C)=1$. Using the rule $(R 2 b)$ it follows quantity $\left(P_{1}\right)=1$. Finally, together with (5) the rule (R6) is fulfilled.

Let $P_{1}=Q_{1}(P, M)$ or $P_{1}=Q_{1}(P, \neg M), \quad P_{2}=$ $Q_{2}(M, S)$ and $P_{3}=Q_{3}(S, P)$ or $P_{3}=Q_{3}(S, \neg P)$. Then $\operatorname{dist}\left(S, P_{2}\right)=\varepsilon$ and $\operatorname{dist}(S, C)=$ quantity $(C)$. Hence using the rule $(R 2 a)$ it follows that quantity $(C)=\varepsilon$ which yields (R6).

\section{Summary and Conclusion}

In the article we continued to study generalized syllogisms using generalized Peterson's rules. We suggested formal definitions of distributivity, quality and quantity rules, respectively. We formally proved that the rules of quantity can inferred from the rules of distirbutivity and quality. The main idea for the future is to propose an algorithm based on generalized Peterson's rules and to analyze syllogisms with $k$-quantifiers and $n$-premises.

\section{Acknowledgement}

The work was supported from ERDF/ESF by the project "Centre for the development of Artificial Inteligence Methods for the Automotive Industry of the region” No. CZ.02.1.01/0.0/0.0/17-049/0008414.

\section{References}

[1] D. Dubois, H. Prade, On fuzzy syllogisms, Comput.Intell. 4 (1988) 171-179.

[2] I. Glöckner, Fuzzy Quantifiers: A Computational Theory, Springer, Berlin, 2006.

[3] P. Hájek, Metamathematics of Fuzzy Logic, Kluwer, Dordrecht, 1998.

[4] P. Lindström, First order predicate logic with generalized quantifiers, Theoria 32 (1966) 186195.

[5] P. Murinová, On modeling of generalized syllogisms with intermediate quantifiers, in: Proc. IFSA-NAFIPS 2019, Lafayette, USA, 2019.
[6] P. Murinová, V. Novák, A formal theory of generalized intermediate syllogisms, Fuzzy Sets and Systems 186 (2013) 47-80.

[7] P. Murinová, V. Novák, Analysis of generalized square of opposition with intermediate quantifiers, Fuzzy Sets and Systems 242 (2014) 89113.

[8] P. Murinová, V. Novák, The structure of generalized intermediate syllogisms, Fuzzy Sets and Systems 247 (2014) 18-37.

[9] P. Murinová, V. Novák, Generalized conjunctive syllogisms with more premisses in fuzzy naturral logic, Uncertainty Modelling in Knowledge Engineering and Decision Making 10 (2016) 282288.

[10] V. Novák, A formal theory of intermediate quantifiers, Fuzzy Sets and Systems 159 (10) (2008) 1229-1246.

[11] M. Pereira-Fariña, F. Díaz-Hermida, A. Bugarín, On the analysis of set-based fuzzy quantified reasoning using classical syllogistics, Fuzzy Sets and Systems 214 (2013) 83-94.

[12] M. Pereira-Fariña, J. C. Vidal, F. Díaz-Hermida, A. Bugarín, A fuzzy syllogistic reasoning schema for generalized quantifiers, Fuzzy Sets and Systems 234 (2014) 79-96.

[13] P. Peterson, Intermediate Quantifiers. Logic, linguistics, and Aristotelian semantics, Ashgate, Aldershot, 2000.

[14] W. D. Ross, Aristotle's Prior and Posterior Analytics, Clarendom Press, Oxford, 1949.

[15] D. G. Schwartz, Dynamic reasoning with qualified syllogisms, Artif.Intell. 93 (1997) 103-167.

[16] B. E. Thompson, Syllogisms using "few", "many" and "most", Notre Dame Journal of Formal Logic 23 (1982) 75-84.

[17] E. Turunen, An algebraic study of peterson's intermediate syllogisms, Soft Computing (2014) 10.1007/s00500-013-1216-2.

[18] L. A. Zadeh, Syllogistic reasoning in fuzzy logic and its applications to usuality and reasoning with dispositions, IEEE Trans. Syst. Man Cybern. 15 (1985) 754-765. 